\title{
Improvement of retrieved FORMOSAT-3/COSMIC electron densities validated by ionospheric sounder measurements at Jicamarca
}

\author{
A. Aragon-Angel, ${ }^{1}$ Y.-A. Liou, ${ }^{2}$ C.-C. Lee, ${ }^{3}$ B. W. Reinisch, ${ }^{4}$ M. Hernández-Pajares, ${ }^{1}$ \\ M. Juan, ${ }^{1}$ and J. Sanz ${ }^{1}$ \\ Received 16 December 2010; revised 4 April 2011; accepted 20 May 2011; published 1 September 2011.
}

[1] Inversion techniques applied to GPS-LEO radio occultation data allow the retrieval of accurate and worldwide-distributed refractivity profiles, which, in the case of the ionosphere, can be converted into electron densities providing information regarding the electron content distribution in this atmospheric region. In order to guarantee the accuracy of the electron density retrievals, two key points should be taken into account: the horizontal gradients of the electronic distribution and the topside electron content above the LEO orbit. The deployment in April 2006 of the satellite Constellation Observing System for Meteorology Ionosphere and Climate (FORMOSAT-3/COSMIC), carrying GPS receivers on board, provides valuable radio occultation data with global and almost uniform coverage overcoming the sparsity of data from previous LEO missions (for instance, GPS/MET, CHAMP, and SAC-C). This is also one of the main limitations of other sources providing direct observations, such as ionosondes. In this study, the improved Abel transform inversion is used to analyze derived ionospheric electron density profiles of the whole year 2007 in a scenario with very high electron density gradients: The neighboring area of Jicamarca $\left(76.9^{\circ} \mathrm{W}, 12^{\circ} \mathrm{S}\right.$, dip latitude: $\left.1^{\circ} \mathrm{N}\right)$, Perú, located at very low latitude and close to the geomagnetic equator, and the influence of the AppletonHartree equatorial anomaly (Davies, 1990). Moreover, different strategies to account for the topside electron content in the occultation data inversion are compared and discussed, taking advantage of the availability of FORMOSAT-3/COSMIC data sets and manually calibrated measurements from Jicamarca DPS. Statistical results show that for the current scenario the improvements are only about $10 \%$, evidencing that the lack of colocation is one important source of error for the classical Abel inversion. Implications with respect to the plasmaspheric contribution have been derived from this data set analysis, in particular, the necessity to account for it specially when the Total Electron Content (TEC) is small.

Citation: Aragon-Angel, A., Y.-A. Liou, C.-C. Lee, B. W. Reinisch, M. Hernández-Pajares, M. Juan, and J. Sanz (2011), Improvement of retrieved FORMOSAT-3/COSMIC electron densities validated by ionospheric sounder measurements at Jicamarca, Radio Sci., 46, RS5001, doi:10.1029/2010RS004578.

\section{Introduction}

[2] Electron density profiles can be derived from radio occultation data. A GPS occultation event occurs when a GPS satellite sets/rises below/above the horizon of a LEO satellite. Under such circumstances, provided the LEO

\footnotetext{
${ }^{1}$ Research Group of Astronomy and Geomatics, Technical University of Catalonia, Barcelona, Spain.

${ }^{2}$ Center for Space and Remote Sensing Research, National Central University, Jhongli, Taiwan.

${ }^{3}$ General Education Center, Ching-Yun University, Jhongli, Taiwan.

${ }^{4}$ Center for Atmospheric Research, University of Massachusetts, Lowell, Massachusetts, USA.

Copyright 2011 by the American Geophysical Union. 0048-6604/11/2010RS004578
}

satellite is equipped with at least one GPS receiver on board and antenna pointing to the limb (which is the case of the FORMOSAT-3/COSMIC constellation [see Rocken et al., 2000]), the change in the delay and the bending of the signal path between the GPS and the LEO satellite caused by the atmosphere can be derived from the observations in the GPS receiver on board the LEO [Hajj and Romans, 1998]. In this study, the basic observable used as main data to retrieve vertical ionospheric profiles is the extra Doppler shift induced by the medium in a single frequency GPS carrier phase [Schreiner et al., 1999]. The new work presented is focused in the analysis of previously studied occultation techniques based on the extra Doppler shift and taking into account the horizontal electron content gradients (improved Abel inverse transform) in a very hard ionospheric scenario: 


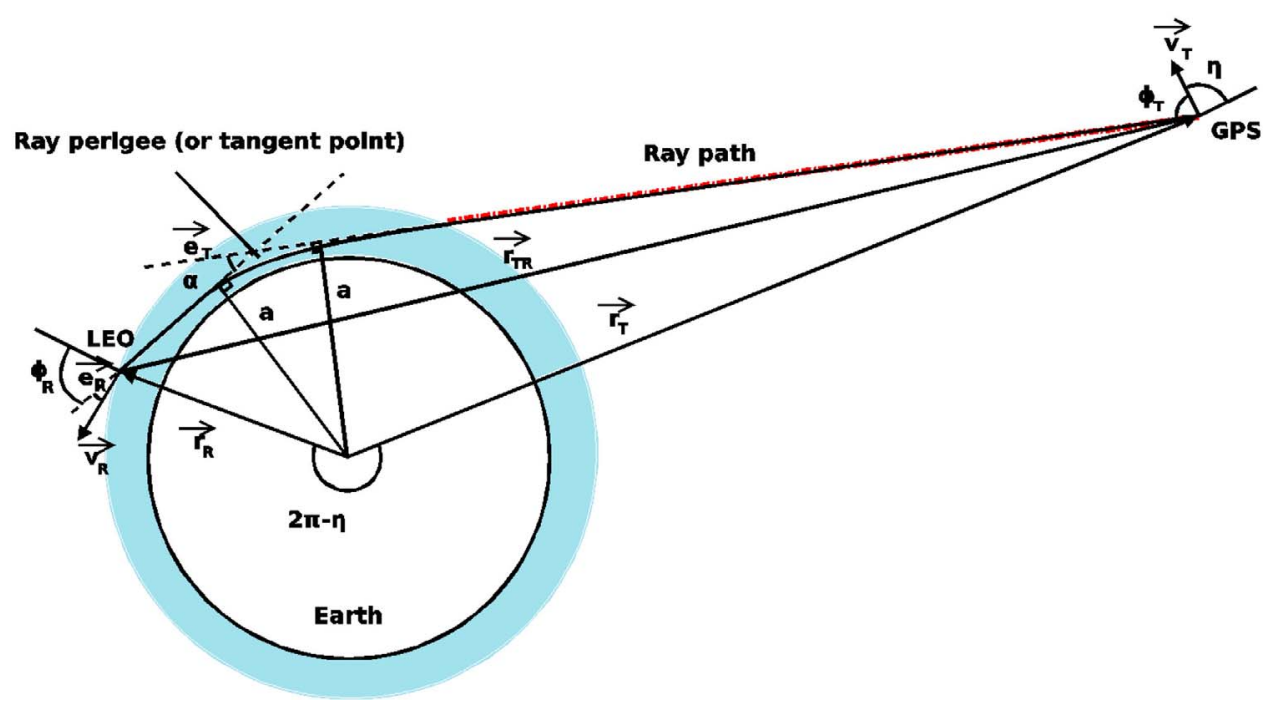

Figure 1. Schematic representation of a radio occultation geometry with parameters involved in equations (2) and (3). In dashed red line, it is represented the part of the ray path for which the electronic contribution is neglected in a first approximation of the inversion procedure.

The neighboring area of Jicamarca, located at $\left(76.9^{\circ} \mathrm{W}\right.$, $12^{\circ} \mathrm{S}$, dip latitude: $1^{\circ} \mathrm{N}$ ), hence exposed to fast variable ionospheric conditions due to its proximity to the geomagnetic equator.

\section{Inversion Methods}

[3] In this paper, electron density profiles have been computed from precise refractivity values retrieved from bending angles derived from the observed excess Doppler frequency shifts in GPS carrier phase L1 [see Hajj and Romans, 1998; Schreiner et al., 1999] in occulting scenarios. The Doppler shift $\left(f_{d}\right)$ of the operating frequency $f_{T}$ is

$$
f_{d}=-\frac{f_{T}}{c} \frac{d L}{d t}
$$

where $L$ stands for $L 1$ carrier phase observable corrected from clock offset drifts, and $c$ is the speed of light in a vacuum. The Doppler shift between the transmitter and the receiver is produced by the atmospheric refractive index change and the relative velocity between transmitter and receiver along the actual signal propagation directions. Due to the change in the refractive index, there is a bending of the signal, which is the excess path that is measured. In order to compute accurate radio occultation inversions, the clock drifts of the GPS transmitter and receiver clocks should be removed from the raw phase data in order to solve the bending angles derived from $L 1$ Doppler phase excess. The Doppler shift of the operating frequency $f_{T}$ can be also derived from

$$
f_{d}=f_{T}\left[\frac{c-n\left(\overrightarrow{r_{R}}\right)\left\|\overrightarrow{v_{R}}\right\| \cos \left(\phi_{R}\right)}{c-n\left(\overrightarrow{r_{T}}\right)\left\|\overrightarrow{v_{T}}\right\| \cos \left(\phi_{T}\right)}-1\right]
$$

where $\overrightarrow{r_{T}}$ and $\overrightarrow{r_{R}}$ represent the position vectors of the transmitter and receiver, $n$ is the refractive index at the specified coordinates, $\overrightarrow{v_{T}}$ and $\overrightarrow{v_{R}}$ are the transmitter and receiver velocities, and the symbols $\phi_{T}$ and $\phi_{R}$ denote the angles between $\overrightarrow{v_{T}}$ and $\hat{e_{T}}$ and $\overrightarrow{v_{R}}$ and $\hat{e_{R}}$, respectively, being $\hat{e_{T}}$ and $\hat{e_{R}}$ the unit vectors tangent to the optical ray path at the transmitter and receiver positions (see Figure 1).

[4] According to Snell's law, the signal path is curved due to the change in the refractive index along the travel path of the signal. Assuming a spherically symmetric medium, Snell's law can be replaced by Bouger's law providing an extra constraint:

$$
n\left(\overrightarrow{r_{T}}\right)\left\|\overrightarrow{r_{T}} \times \hat{k_{T}}\right\|=n\left(\overrightarrow{r_{R}}\right)\left\|\overrightarrow{r_{R}} \times \hat{k_{R}}\right\|
$$

where $\hat{k_{T}}$ and $\hat{k_{R}}$ are the unit vectors in the direction of the straight line connecting the transmitter to the receiver (see Figure 1). Equations (2) and (3) need to be solved simultaneously to obtain the total atmospheric bending $(\alpha)$, i.e., the bending of the actual ray path with respect the straight line propagation, which is explicated in Figure 1. Nevertheless, $n$ at $\overrightarrow{r_{T}}$ and $\overrightarrow{r_{R}}$ are unknown. One first approximation to the problem is to consider that there is no electron content above the LEO orbit which, for the case of working with the Doppler shift, implies that the gradient of the refractive index above the LEO position remains equal to one at the LEO height.

[5] This approximation overestimates the electron density not more than $0.5 \%$ [Hajj and Romans, 1998]. In fact, the higher the altitude of the LEO, the more reasonable it becomes.

\subsection{The Classical Abel Inversion}

[6] In a spherical symmetric medium, the bending of the signal can be related to the refractive index by means of the following integral [Fjeldbo et al., 1971]:

$$
\alpha(a)=2 a \int_{0}^{\infty} \frac{1}{\sqrt{a^{\prime 2}-a^{2}}} \frac{d \ln (n)}{d a^{\prime}} d a^{\prime}
$$



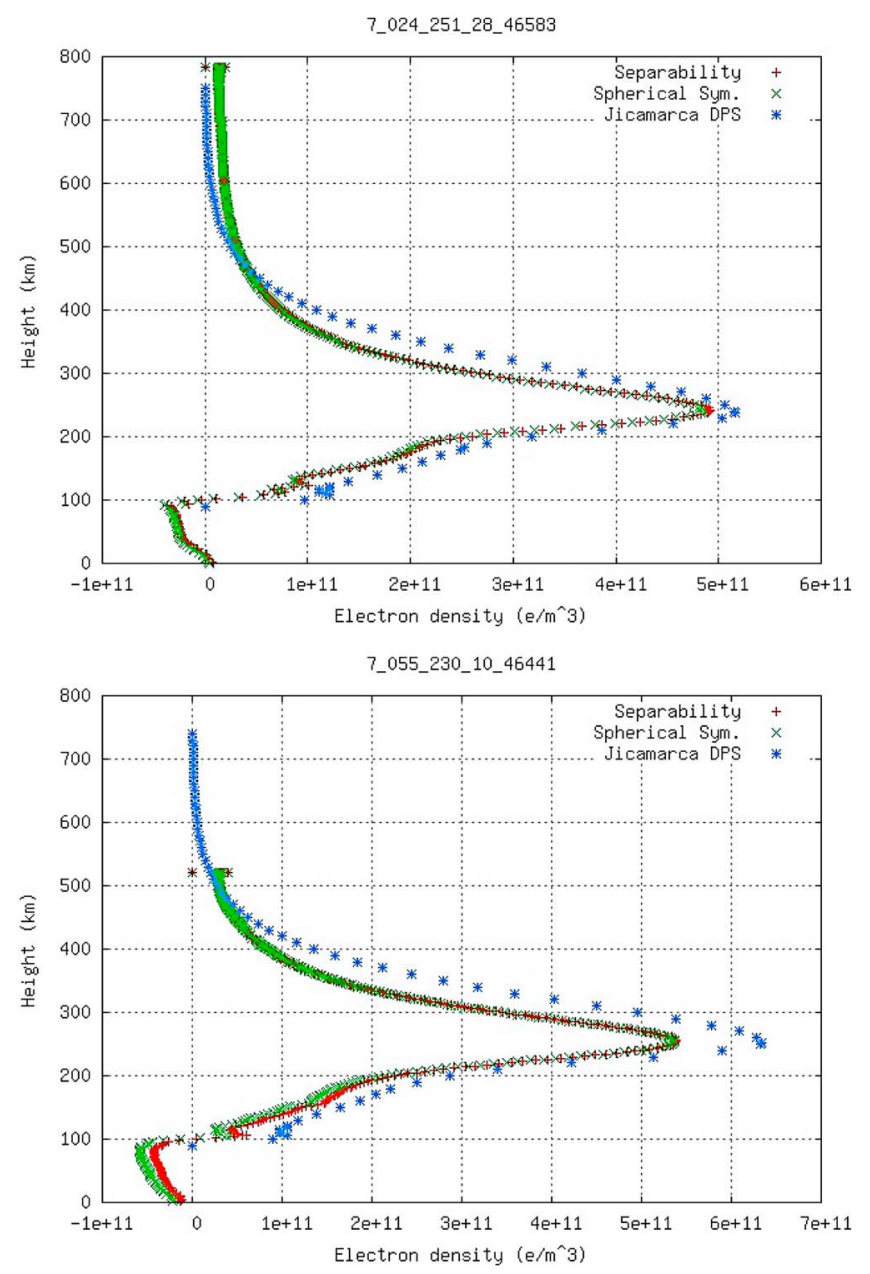

Figure 2. Examples of electron density profiles derived from FORMOSAT-3/COSMIC data versus colocated Jicamarca digisonde measurements. These series belong to the whole year 2007 intercomparison with no upper ionospheric contribution considered.

where $\alpha$ stands for the bending angle and $a$, for the impact parameter, i.e., the closest point to the Earth center along the optical ray path (see Figure 1). By using an Abel integral inverse transform, equation (4) can be inverted [see Tricomi, 1985], obtaining the refractive index as a function of the impact parameter $a$

$$
\ln (n(a))=\frac{1}{\pi} \int_{a}^{\infty} \frac{\alpha\left(a^{\prime}\right)}{\sqrt{a^{\prime 2}-a^{2}}} d a^{\prime}
$$

[7] The evaluation of the integral of equation (5) in the upper limit requires the knowledge of the bending $\alpha$ to the furthest limits of the atmosphere. For practical reasons, the bending angles above the LEO position (typically small for heights above $700 \mathrm{~km}$ ) can either be neglected, somehow extrapolated or replaced by a climatological model [Schreiner et al., 1999]. In the current study, firstly, this integral is solved up to the LEO height, neglecting the contribution of the bending angles above the LEO orbit. Second, three different approaches to account for the upper electronic contribution are discussed.

\subsection{The Improved Abel Inversion}

[8] The classical Abel inversion mainly presents two mismodelings, such as the spherical symmetry assumption and the neglect of the topside electron content contribution. The former can be mitigated by means of the separability concept [Hernández-Pajares et al., 1998, 2000]. The latter is going to be discussed in the next section. It is believed that the dominant error is due to the spherical symmetry assumption imposed on the ionosphere [see Wu et al., 2009].

[9] The separability concept can be integrated in the inversion procedure by considering not only the radial component $(\mathrm{H})$ dependence but also longitude and latitude in the density calculation. Therefore, the electron density, $N_{e}$, can be reformulated as

$$
N_{e}(L T, L A T, H)=\operatorname{VTEC}(L T, L A T) \cdot F(H)
$$

where $V T E C(L T, L A T)$ represents the Vertical Total Electron Content $(V T E C)$ at $(L T, L A T)$ location and $F(H)$ stands for the so-called shape function, which assumes the height dependence. It is a simple but very effective approach: VTEC can provide a real ground-based proxy for the horizontal variability of the electron density $N_{e}$ (under the assumption that it is the same at every height in the occultation region). The new estimated value is the shape function $F$ instead of the electron density. Indeed, when considering the latidudinal and longitudinal variation of VTEC into the electron density, the quality of the retrieved electron density profiles is improved. The benefits of implementing the separability hypothesis to bending angles were already stated with respect to actual ionosonde data [see Aragon-Angel et al., 2009]. These comparisons confirmed a global improvement in the critical frequency estimations of the $F 2$ peak of $45 \%$ in Solar Minimum, whereas in the E layer, the improvement in electron density estimates reached up to $32 \%$.

\section{Upper Ionospheric Contribution}

[10] In this section, the contribution of the electron content above the LEO orbit in the inversion process is tackled (see Figure 1). One first approximation to the problem is to consider that there is no significant electron content above the LEO orbit (see example in Figure 2).

[11] Nevertheless, this upper electron contribution should be accounted for in a simple but accurate way in order to further improve the inversion of the bending angles derived from the GPS observations tracked by the LEO satellite. Although this issue may not be critical for LEOs with nominal orbits above $700 \mathrm{~km}$, it is a crucial point to be considered with lower Earth orbiters. For instance, within the FORMOSAT-3/ COSMIC constellation, not all six satellites have been at the same nominal altitude at all times, since, after their launch, some of them remained in a parking orbit (at about $500 \mathrm{~km}$ ) while the others were reaching their final destination orbit (at about $800 \mathrm{~km}$ ). Thus the lower the LEO altitude, the bigger the mismodeling error introduced by neglecting the LEO topside electron content.In the following subsections, 

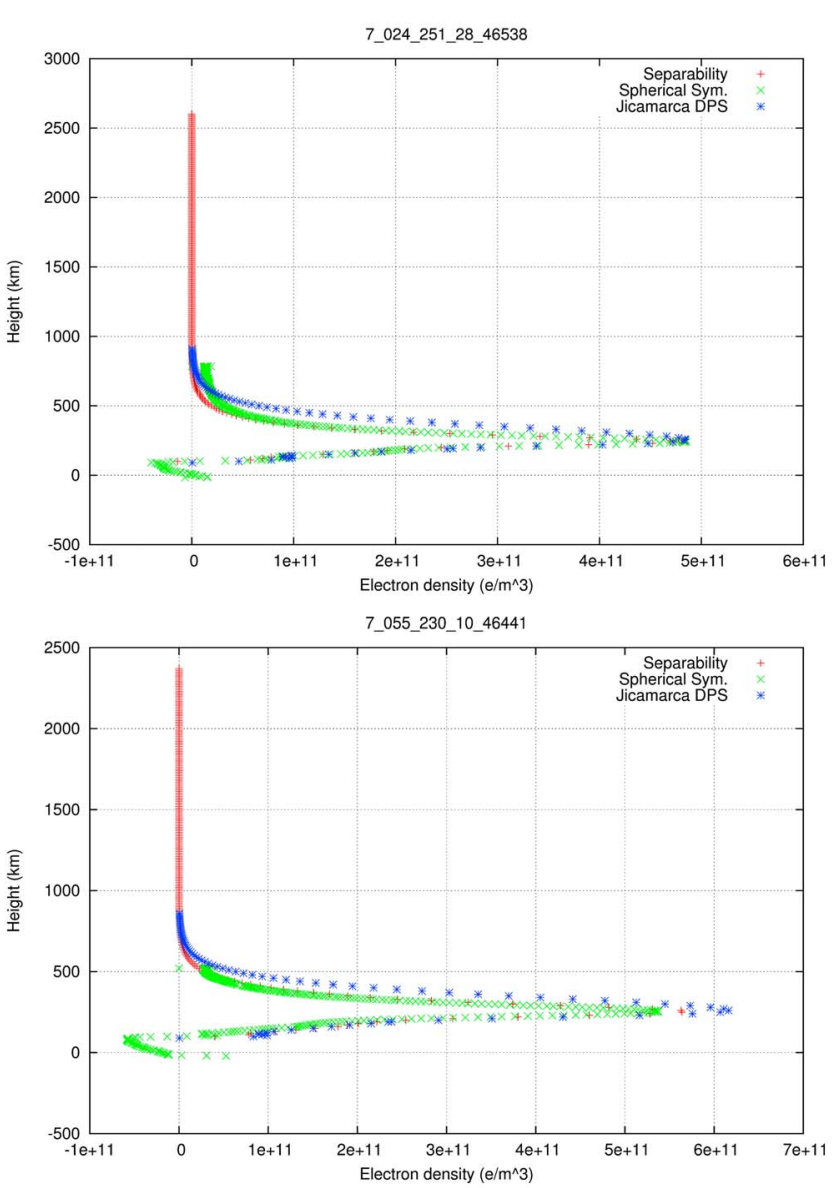

Figure 3. Example of extrapolated profile versus colocated Jicamarca digisonde measurements, corresponding to the profiles shown in Figure 2.

two approaches to overcome this issue are going to be proposed: Firstly, the usage of a climatological model, and second, an exponential decay.

\subsection{First Approach: Climatological Model}

[12] A possible solution to the upper ionosphere and plasmasphere issue was found by Jakowski et al. [2002, 2003], where the inversion was assisted with a "first" guess extracted by a Chapman layer model for the topside ionosphere. In this study, the NeQuick model is going to be used in order to provide the first value of electron density at the starting point of the radio occultation, which will be conveniently transformed into the corresponding refractive index value.

[13] Procedure: Considering the actual geometry and epoch of the occultations (i.e., real line-of-sights), the NeQuick model is run for the upper observation (the one corresponding to the highest impact parameter) and the electron density for such point is obtained. Once this initial value is calculated, the inversion is performed by means of both, classical Abel and Improved Abel inversions and the global profiles of electron density are retrieved.

\subsection{Second Approach: Exponential Decay}

[14] Another approach to the problem of determining the upper contribution of the ionosphere to the derived profiles would be by performing an exponential extrapolation of the profile as done by Hernández-Pajares et al. [2000] or, alternatively, in the input data as by Hajj and Romans [1998]. According to Schreiner et al. [1999], an exponential extrapolation approach is more appropriate than the use of a climatological model. For the current study, the retrieved density profiles have been extrapolated by means of an exponential decay for the shape function $F(H)$ :

$$
F(H)=A \cdot e^{-\frac{H}{h_{T}}}+B
$$

where $A$ is a scaling factor, $h_{T}$ stands for the scale height and is the factor that controls the rate of decrease and, $B$ stands for a bias. In principle, since the electron density has to become zero when the height $H$ tends to infinite (the same applies for the shape function via equation (6)), the constant $B$ should be subtracted from the original profile and also the exponential fit to guarantee that the resulting shape function has a vertical asymptote at $N_{e}=0$ (consequently, at $F=0$ ). This bias $B$ is thought to take into account the fact that the topside (protonospheric contribution) is basically constant and would not contribute to the bending since it is based on increments of refractive index. Without the consideration of $B$ in equation (7), only a determination of $\Delta N e$ would be obtained, since there is an ambiguity in the initial value, that should be solved with the use of one of the methods presented in this section. As first trial, three equidistant points from the original profile (located above the F2-layer peak) were used to perform the extrapolation. Nevertheless, due to sudden changes in convexity/concavity of the profiles, the lack of data or abrupt jumps, the success of the extrapolation was not guaranteed for a reasonable number of profiles. For this reason, the strategy was made more robust by performing several extrapolations to the same profile using different triad of points to anchor the extrapolation. Therefore, different sets of parameters $A, h_{T}$ and $B$ would be obtained from each of these extrapolations. By setting a tolerance (RMS), $A, h_{T}$ and $B$ would be statistically best fitted into one final solution for the given initial profile. An example of the extrapolated profile versus the corresponding original one and an external reference are provided in Figure 3.

\section{Experiment: FORMOSAT-3/COSMIC Versus Jicamarca DPS}

[15] In order to conduct the intercomparisons between results obtained with the two former approaches for the upper electron content contribution, a reliable reference source for electron densities was required. In this sense, manually supervised Digisonde Portable Sounder (DPS) measurements at Jicamarca $\left(76.9^{\circ} \mathrm{W}, 12^{\circ} \mathrm{S}\right)$ were available for the whole year 2007 with a sample rate of $15 \mathrm{~min}$. This high-quality real data offers a valuable possibility to perform the proof of concept and the comparison of the inverted electron density profiles using the presented approaches for the upper ionosphere estimation. Moreover, there is practically no colocation error with respect location since the ionospheric profiles from Jicamarca DPS have been compared with extremely close colocated FORMOSAT-3/COSMIC profiles: Only when the tangent points of the occultation ray paths at the F2-peak are located in the range of $9-15^{\circ} \mathrm{S}$ and $73.9-79.9^{\circ} \mathrm{W}$, then the FORMOSAT-3/COSMIC profile is 


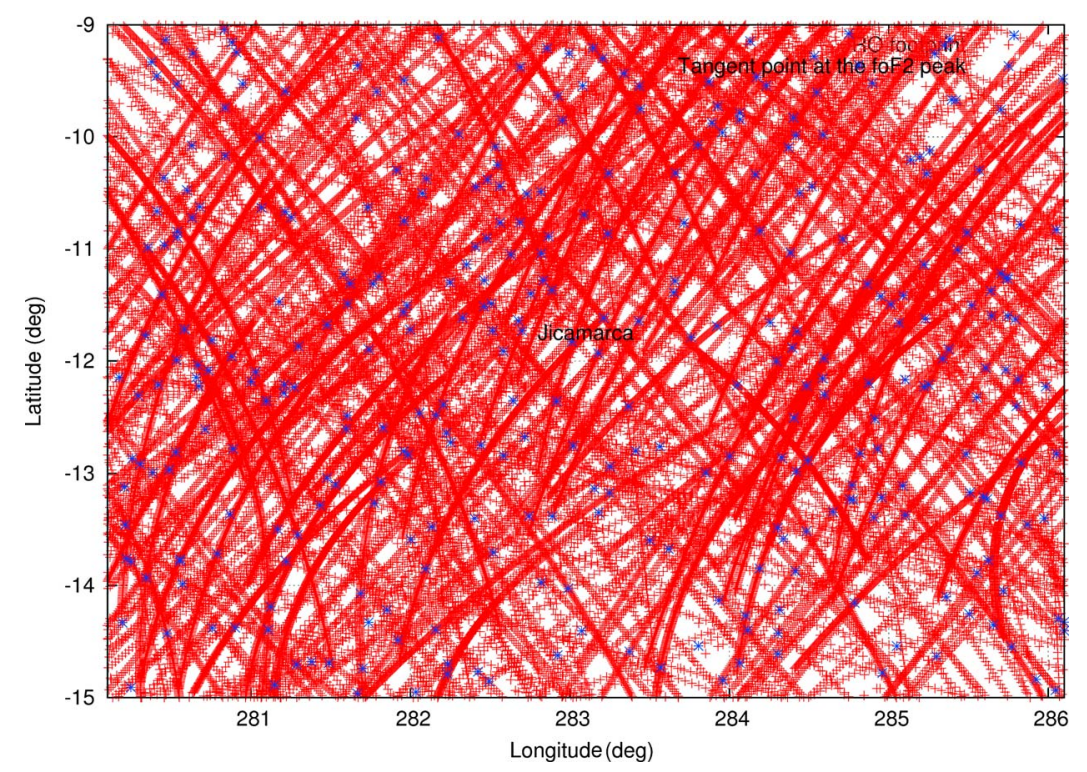

Figure 4. In red, footprints of FORMOSAT-3/COSMIC radio occultation data corresponding to the selected profiles. In blue, tangent points at the F2-peak height of each selected profiles. Jicamarca DPS $\left(76.9^{\circ} \mathrm{W}, 12^{\circ} \mathrm{S}\right.$, dip latitude: $\left.1^{\circ} \mathrm{N}\right)$ is located in the center of the lattice.

selected to be compared with the corresponding digisonde profile (see Figure 4). Regarding temporal colocation, a time span of $15 \mathrm{~min}$ has been considered around the time of occurrence of the radio occultation to allow comparisons with Jicamarca DPS measurements. Three consequences of this tight colocation are derived: (1) There is relatively small spatial mismatch error with respect to the truth to compare. (2) There is a temporal mismatch error limited by the $15 \mathrm{~min}$ colocation with respect to the truth to compare. (3) Since the truth comes from calibrated real data, this fact will help assessing the performances under real circumstances.

[16] Consequently, since the most important sources of errors are highly mitigated in this experiment, this study will give an overview of the goodness of each approach itself (i.e., the best performance one can expect from each of them).

[17] The experiment data correspond to a 1 year period, 2007 (222 highly colocated occultations have been deeply studied). GPS raw data from FORMOSAT-3/COSMIC satellites (CDAAC level 0 products) were processed with the aid of the global VTEC maps computed for 2007 by the Technical University of Catalonia (UPC), and distributed in IONEX format by the International GNSS Service (IGS). These maps provide the required information of the horizontal variation of the electron density to perform improved Abel inversion.

[18] In order to rule out doubtful intercomparisons, the slab thickness $(\tau)$ parameter has been used to detect and remove potential outliers of electron density values (only $\tau$ belonging to the $[150,1600](\mathrm{km})$ interval have been considered; see Figure 6, bottom). For the ionosonde measurements, it is calculated combining the corresponding VTEC information with the ionosonde derived $F 2$ layer peak electron density, $N m F 2$ :

$$
\tau=\frac{V T E C}{N m F 2}
$$

For a solved occultation, it is also possible to assign a slab thickness value recalling the expression in equation (6):

$$
\tau=\frac{V T E C}{N m F 2}=\frac{V T E C}{V T E C \cdot F(h m F 2)}=\frac{1}{F(h m F 2)}
$$

\section{Results: The foF2 Comparisons With Jicamarca Measurements}

[19] All the statistics presented in the forthcoming tables take into account the local time of the measurement since the ionospheric variability at Jicamarca is extremely different with respect to the local time (see Figure 5). At Jicamarca location: (1) Daytime from 06:00 to 19:00 LT (11:00-23:00 UT). (2) Nighttime from 22:00 to 06:00 LT (03:00-10:00 UT).

[20] These local times have determined the three time spans for which the statistics have been divided into (day, night and, dusk and dawn), apart from a global comparison providing bias, $\sigma$ (both expressed in $\mathrm{MHz}$ ) and relative RMS difference (in percentage) that gives an overview of
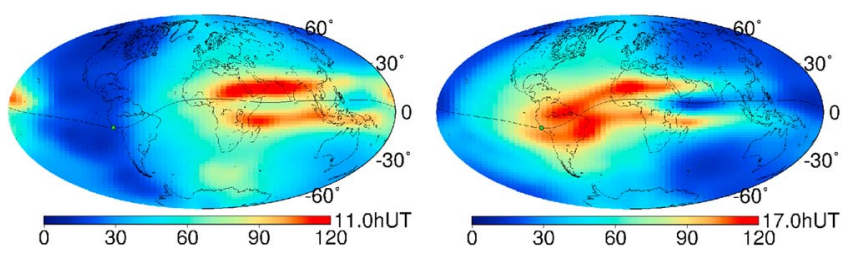

Figure 5. Example of the variability of the electronic distribution around Jicamarca location (marked with a green point): two Global Ionospheric Maps in IONEX format computed and distributed by UPC for 16 May 2007. Maps corresponding to (left) 11:00 UT and (right) 17:00 UT. 

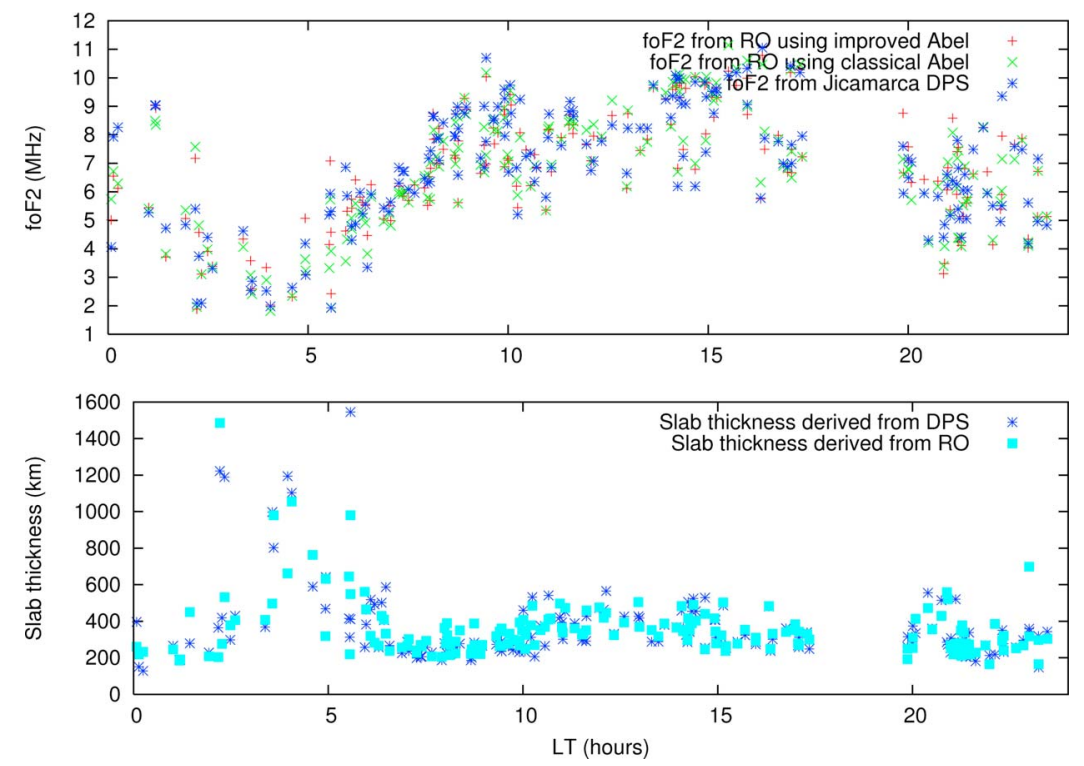

Figure 6. (top) Dispersion of the critical frequency of the $F 2$ layer versus local time throughout 2007 at the neighborhood of Jicamarca: In red, $f o F 2$ derived from FORMOSAT-3/COSMIC radio occultations by means of the improved Abel inversion. In green, $f o F 2$ derived from FORMOSAT-3/COSMIC radio occultations by means of the classical Abel inversion. In blue, Jicamarca DPS $f o F 2$ measurements. (bottom) Dispersion of the slab thickness versus local time throughout 2007 at the neighborhood of Jicamarca: In light blue, slab thickness values derived from radio occultation inverted profiles. In dark blue, slab thickness values derived from Jicamarca DPS measurements.

the performance for each methodology with respect the reference DPS measurements.

[21] In Figure 6, top, it is shown the consistency for all year 2007 between $f o F 2$ measurements from ionosonde and derived from inverted radio occultation measurements (with both, classical (green) and improved (red) Abel inversions). Note the dependency on the local time: There is a marked valley around 04:00 LT and, all derived and measured foF2 follow the same variation pattern along the year. Also notice (Figure 6, bottom) the opposite behavior of the slab thickness and $f_{o F} 2$ dependency with respect to local time around 04:00 LT.

[22] In the next sections, the statistical results corresponding to this data set while comparing the proposed extrapolation approaches are presented. It should be pointed out that, although in previous studies (for instance, AragonAngel et al. [2009]), the general improvement by implementing

Table 1. Neglected Upper Contribution for Topside Modeling: Global Comparisons of $F 2$ Layer Critical Frequency Discrepancies With Ionosondes for Colocated Radio Occultations With Jicamarca DPS, Bending Angles $(\alpha)$ Using Separability Versus Classical Abel Inversion ${ }^{\mathrm{a}}$

\begin{tabular}{lccc}
\hline & & $\begin{array}{c}\text { Improved Abel } \\
\text { Inversion } \\
\text { Bias } \pm \sigma \\
\text { [Rel. RMS \%] }\end{array}$ & $\begin{array}{c}\text { Classical Abel } \\
\text { Inversion } \\
\text { Bias } \pm \sigma \\
\text { [Rel. RMS \%] }\end{array}$ \\
\hline Day & 105 & $-0.20 \pm 0.42[6.3]$ & $-0.13 \pm 0.45[6.4]$ \\
D\&D & 25 & $-0.14 \pm 0.52[9.5]$ & $0.09 \pm 0.56[10.0]$ \\
Night & 15 & $0.01 \pm 0.41[7.5]$ & $-0.13 \pm 0.63[12.0]$ \\
Global results & 145 & $-0.13 \pm 0.46[7.0]$ & $-0.13 \pm 0.48[7.6]$ \\
\hline
\end{tabular}

${ }^{\mathrm{a}}$ Bias and $\sigma$ are expressed in MHz. D\&D stands for dusk and dawn. separability could reach up to $45 \%$, in the current scenario, where the reference is highly colocated with the retrieved profiles, the improvement is only about $10 \%$ evidencing the fact that the lack of colocation is one important source of error for the classical Abel inversion. The shape function $F$ obtained when applying separability allows to spread the intrinsic information of each profile wider than the electron density profile itself: it would be possible to build a new electron density in the surrounding area of the occultation by multiplying $F$ by the $V T E C$ of the new longitudes and latitudes. In this way, new density profiles would be obtained extended from the previous one via the information contained within the shape function which is considered constant.

\subsection{No Upper Ionospheric Contribution}

[23] Table 1 summarizes the comparisons of the critical frequency of the $F 2$ peak layer when neglecting the upper

Table 2. Climatological Model for Topside Modeling: Global Comparisons of F2 Layer Critical Frequency Discrepancies With Ionosondes for Colocated Radio Occultations With Jicamarca DPS: Bending Angles $(\alpha)$ Using Separability Versus Classical Abel Inversion ${ }^{\mathrm{a}}$

\begin{tabular}{lccc}
\hline & & $\begin{array}{c}\text { Improved Abel } \\
\text { Inversion } \\
\text { Bias } \pm \sigma \\
\text { [Rel. RMS \%] }\end{array}$ & $\begin{array}{c}\text { Classical Abel } \\
\text { Inversion } \\
\text { Bias } \pm \sigma \\
\text { [Rel. RMS \%] }\end{array}$ \\
\hline Day & 95 & $-0.07 \pm 0.44[6.0]$ & $0.01 \pm 0.48[6.5]$ \\
D\&D & 22 & $0.01 \pm 0.47[8.4]$ & $-0.08 \pm 0.61[11.1]$ \\
Night & 18 & $0.06 \pm 0.43[8.5]$ & $-0.03 \pm 0.59[11.5]$ \\
Global results & 135 & $-0.04 \pm 0.45[6.6]$ & $-0.01 \pm 0.52[7.7]$ \\
\hline
\end{tabular}

${ }^{\mathrm{a}}$ Bias and $\sigma$ are expressed in MHz. D\&D stands for dusk and dawn. 
Table 3. Exponential Decay for Topside Modeling: Global Comparisons of F2 Layer Critical Frequency Discrepancies With Ionosondes for Colocated Radio Occultations With Jicamarca DPS: Bending Angles $(\alpha)$ Using Separability Versus Classical Abel Inversion $^{\mathrm{a}}$

\begin{tabular}{lccc}
\hline & $\begin{array}{c}\text { Improved Abel } \\
\text { Inversion } \\
\text { Bias } \pm \sigma \\
\text { [Rel. RMS \%] }\end{array}$ & $\begin{array}{c}\text { Classical Abel } \\
\text { Inversion } \\
\text { Bias } \pm \sigma \\
\text { [Rel. RMS \%] }\end{array}$ \\
\hline Day & Nr. Comp. & $-0.21 \pm 0.42[6.5]$ & $-0.14 \pm 0.43[6.4]$ \\
D\&D & 13 & $-0.01 \pm 0.54[9.6]$ & $-0.16 \pm 0.49[9.1]$ \\
Night & 11 & $0.19 \pm 0.37[7.9]$ & $0.13 \pm 0.53[10.4]$ \\
Global & 108 & $-0.15 \pm 0.45[7.0]$ & $-0.12 \pm 0.47[7.0]$ \\
\hline
\end{tabular}

${ }^{a}$ RMS threshold set to $4 e^{-8}$. Bias and $\sigma$ are expressed in MHz. D\&D stands for dusk and dawn.

electron content above the LEO orbit. In all cases, the improved Abel transform inversion improves the results of the classical Abel inversion, specially during local night time. There is a negative bias always present in the classical Abel inversion results.

\subsection{Climatological Model for Upper Ionospheric Contribution}

[24] Table 2 summarizes the $F 2$ critical frequency comparisons when the initial guess for the refractive index is provided by NeQuick: The peak of the electron density (i.e., $N m F 2$ ) is extracted from each of these new profiles, transformed into frequency and compared with the colocated values provided by Jicamarca DPS. These results can be also directly compared with the original inversion presented in section 2.2 with the a priori assumption of no upper contribution from the ionosphere above the LEO position. In all cases, the Abel transform inversion using separability improves the results of the classical Abel inversion. In Table 2, there is a slight improvement regarding results in Table 1 during daytime and dusk/dawn, although they worsen during nighttime for separability. The opposite behavior is found for spherical symmetry results. The negative bias always present in the classical Abel inversion results has now been compensated.

\subsection{Extrapolation Scheme for Upper Ionospheric Contribution}

[25] Table 3 summarizes the $F 2$ critical frequency comparisons when an extrapolation scheme is used for the first guess of the refractive index. In all cases, the improved Abel transform inversion improves the results of the classical Abel inversion. In general, it has been noticed worse performances in the classical Abel when becoming more restrictive with the tolerance (RMS) associated to the extrapolation scheme (see section 3.2).

\subsection{Local Effects on Ne Profiles}

[26] While studying the data sets from this experiment, all derived electron density profiles were plotted against their corresponding colocated DPS measurements. In such plots, all diurnal profiles from the DPS systematically showed potbellied topsides, which are calculated through models, in spite of the agreement shown between foF 2 values from FORMOSAT-3/COSMIC versus Jicamarca DPS in Tables 1, 2 , and 3 . For this reason, the difference between these profiles were represented filtering by daytime and nighttime to analyze such effect. In principle, observing Figure 7, a clear underestimation of the topside of the electron density profiles during local daytime for FORMOSAT-3/COSMIC data would be derived while compared to the DPS topside. Nevertheless, since the DPS only provides measurements up to the $F 2$ layer peak, this would basically imply that the DPS topside model

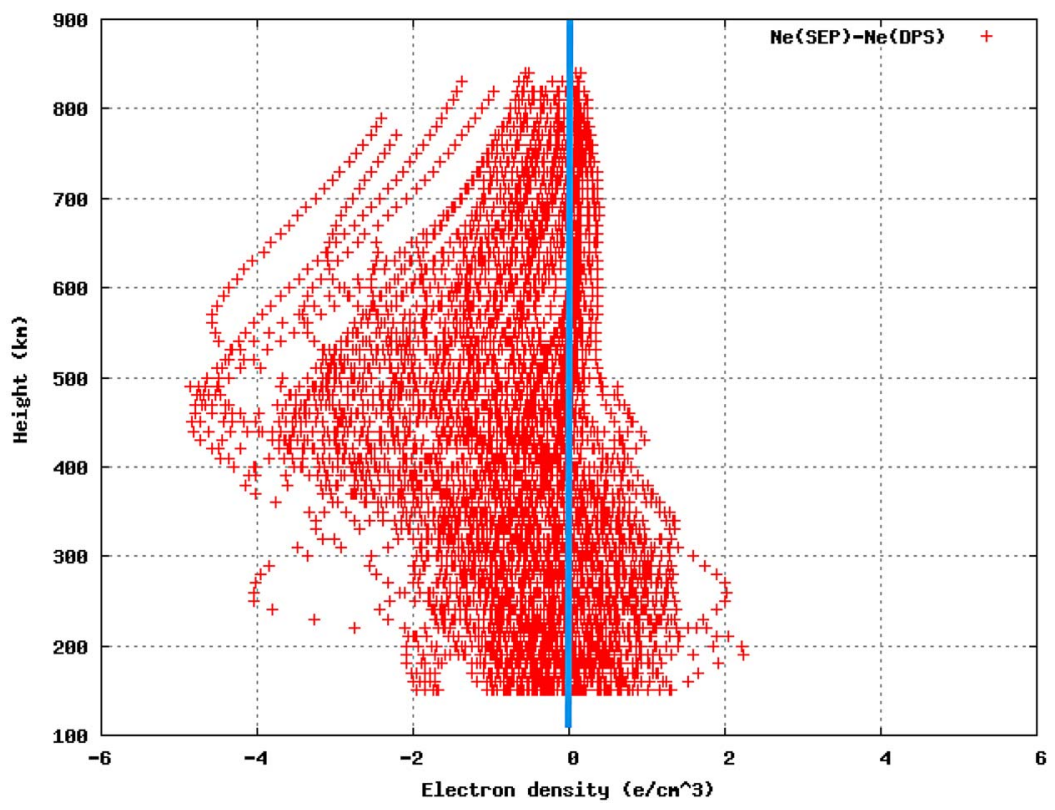

Figure 7. Local effect on NmF2: Day. The difference of electron densities from every FORMOSAT-3/ COSMIC profile derived using separability and the corresponding DPS profile is depicted here. 


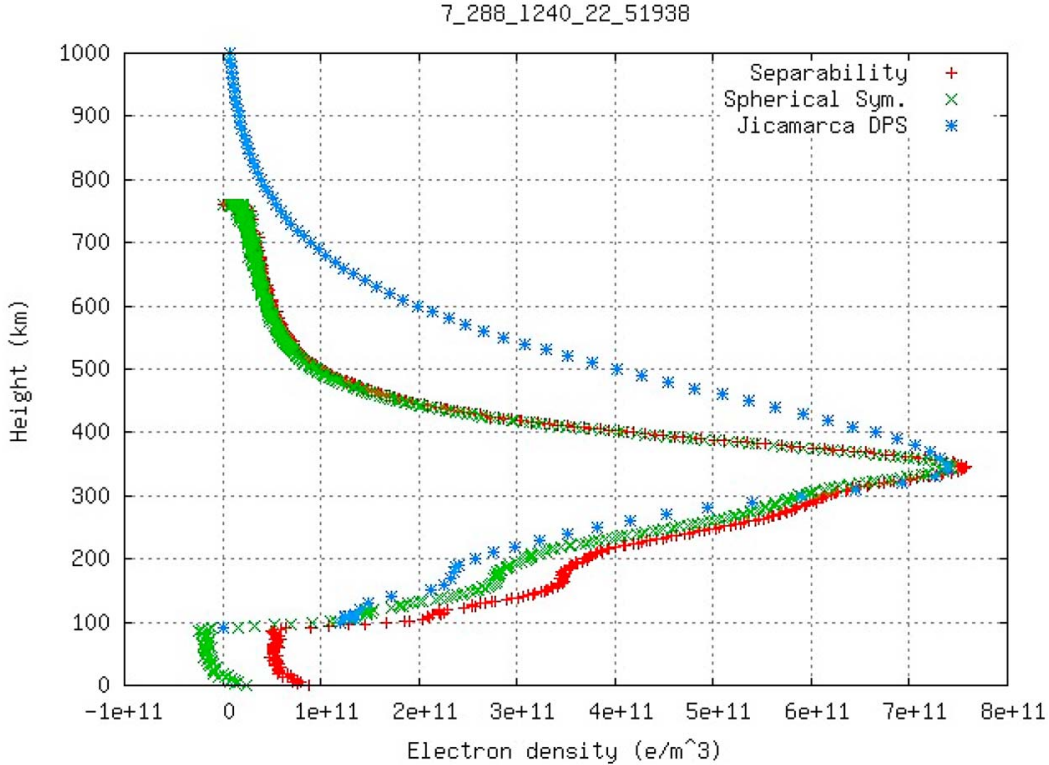

Figure 8. Example of the local effect on NmF2: Day. Notice the potbellied topside from Jicamarca DPS data while compared to FORMOSAT-3/COSMIC derived profiles.

for the electron content overestimates it during local day at latitudes such as Jicamarca's. A typical example corresponding to local day at Jicamarca is provided in Figure 8. Looking at Figure 9, corresponding to local night profiles, an even distribution of the differences between the electron density profiles derived from FORMOSAT-3/COSMIC data and Jicamarca DPS measurements is found. Recalling the fact that the breadth of the density profiles is directly related to the slab thickness, a revision would be suggested in the used models for the diurnal low-latitude topside
DPS modeling. Efforts are already made in this direction, for instance, the use of IMAGE radio plasma imager (RPI) measurements to develop an empirical electron density profile model from the $F 2$ peak to several Earth radii [Reinisch et al., 2008].

\section{Validity of the Separability Hypothesis: Using the Separability Nature of the Electron Density}

[27] The fact that the electron density can be expressed by means of a shape function $F$ assuming the height dependency

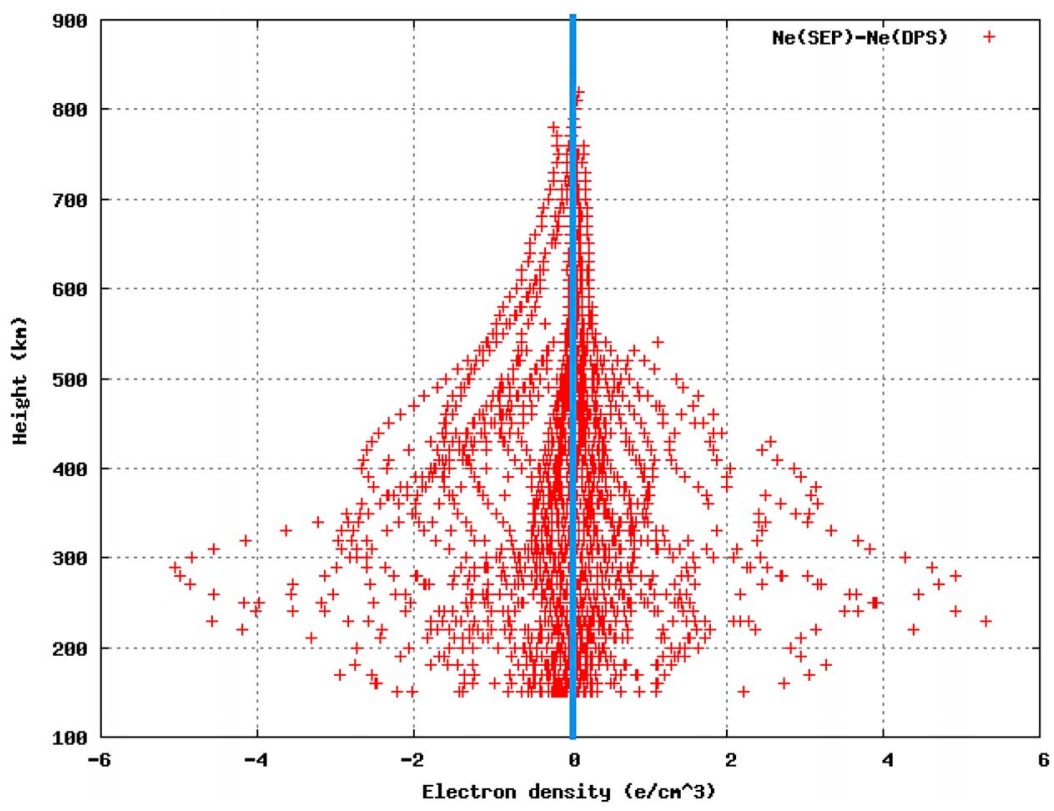

Figure 9. Local effect on NmF2: Night. The difference of electron densities from every FORMOSAT-3/ COSMIC profile derived using separability and the corresponding DPS profile is depicted here. 


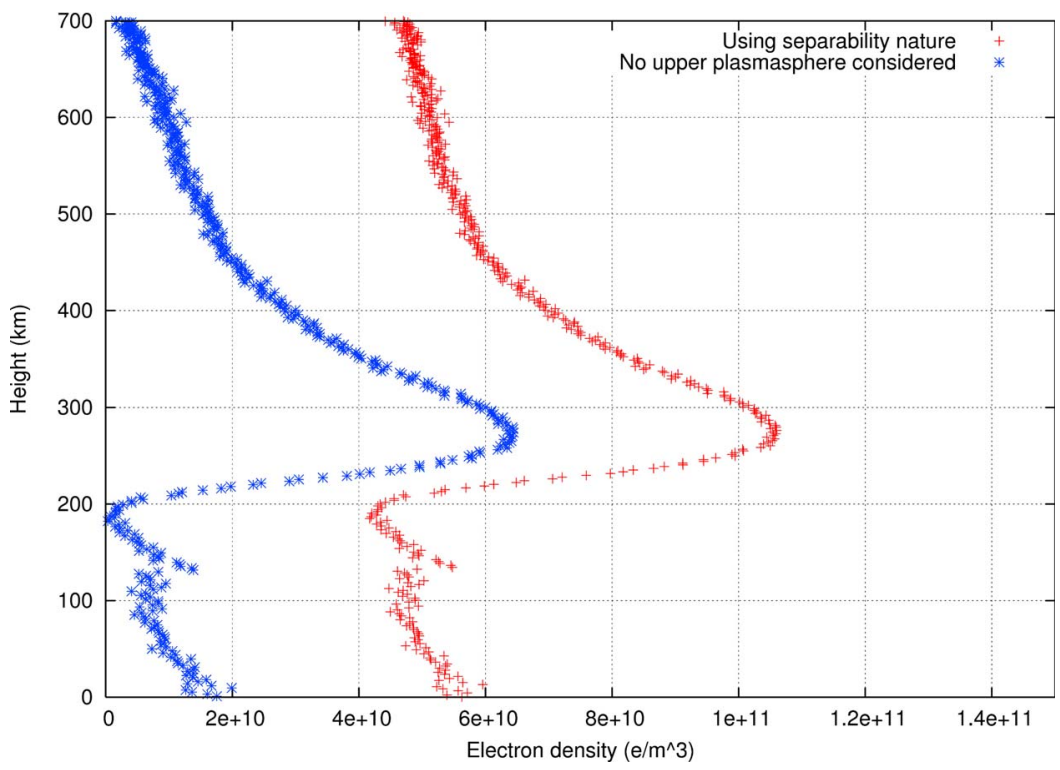

Figure 10. Example of profile solved considering no upper ionospheric contribution (blue) versus using the nature of separability via equation (10) (red).

and the VTEC associated to the corresponding horizontal coordinates as reflected in equation (6), implicitly means that the integral value of the shape function along the vertical should be 1 :

$$
\int F(h) d h=1
$$

This integral expression can be discretized, hence becoming

$$
\sum F(h) \Delta h=1
$$

It should be pointed out that, if no scheme for the upper plasmaspheric contribution is used, some error is introduced (it could reach up to several TECU). When using separability by means of equation (6), the unknown to be solved is $F$ and, actually, when working with the bending angle, the solution to the system is not $F$ but $\Delta F$, that can be solved recursively after providing an initial value of $F$ at the highest height. Taking into account that $\Delta F$ is a discrete function, consecutive differences of $F$ values can expressed as

$$
\Delta F_{i}=F\left(h_{i+1}\right)-F\left(h_{i}\right)
$$

assuming in this expression that $h_{1}>h_{2}>\ldots>h_{n}$ being $n$ the total number of input data. Therefore, the actual $F\left(h_{i}\right)$ values can be derived from the knowledge of $\Delta F$ and the initial $F\left(h_{1}\right)$ :

$$
F\left(h_{i}\right)=F\left(h_{i-1}\right)+\Delta F_{i-1}=F\left(h_{1}\right)+\sum_{k=1}^{i-1} \Delta F_{k}
$$

Using this expression for each $F\left(h_{i}\right)$ and replacing them into equation (11) will lead to

$$
\begin{aligned}
& F\left(h_{1}\right)\left(h_{1}-h_{2}\right)+F\left(h_{1}\right)\left(h_{2}-h_{3}\right)+\Delta F_{1}\left(h_{2}-h_{3}\right) \\
& \quad+F\left(h_{1}\right)\left(h_{3}-h_{4}\right)+\Delta F_{1}\left(h_{3}-h_{4}\right)+\Delta F_{2}\left(h_{3}-h_{4}\right) \\
& \quad+\ldots F\left(h_{1}\right)\left(h_{n-1}-h_{n}\right)+\Delta F_{1}\left(h_{n-1}-h_{n}\right)+\ldots \\
& \quad+\Delta F_{n-2}\left(h_{n-1}-h_{n}\right)=1
\end{aligned}
$$

where consecutive terms with opposite signs will be canceled out, remaining only the ones appearing in the following expression:

$$
F\left(h_{1}\right)\left(h_{1}-h_{n}\right)+\Delta F_{1}\left(h_{2}-h_{n}\right)+\ldots+\Delta F_{n-2}\left(h_{n-1}-h_{n}\right)=1
$$

Hence, the $F\left(h_{1}\right)$ value can be deduced:

$$
F\left(h_{1}\right)=\frac{1}{h_{1}-h_{n}}\left[1-\sum_{i=1}^{n-2} \Delta F_{i}\left(h_{i+1}-h_{n}\right)\right]
$$

obtaining a first guess to solve the recursive system. Applying the constraint given by equation (16) to solve the inversion problem with the same data set used in the previous experiments, the statistical results showed no agreement with digisonde data. In Figure 10, it is depicted the resulting profile using equation (16) compared with the previously solved density profile when considering no upper ionospheric contribution. The new profile (red) is far to the right, meaning that the initial value for the shape function was too big. This red profile would lead to unrealistic TEC values associated to the profile when integrated. The conclusion is that the integral value of the shape function is sometimes smaller than one as assumed in equation (10). At this point, we questioned how the distribution of the integral values of the shape functions 


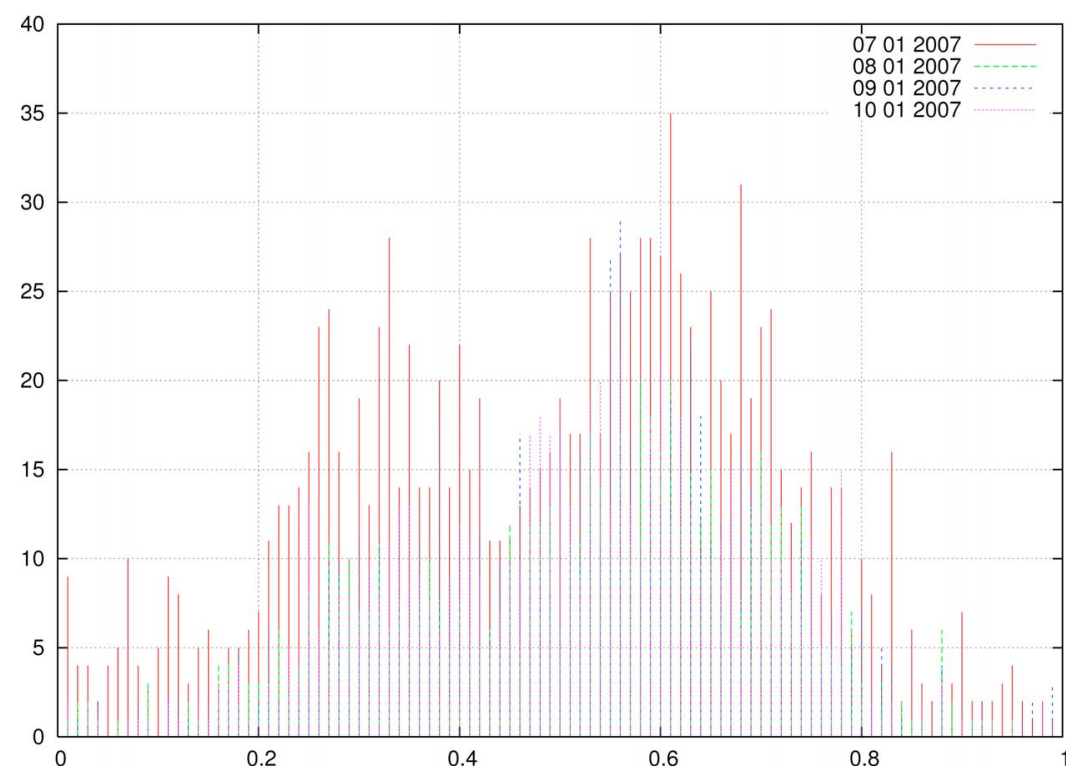

Figure 11. Histogram of the values of the integral of the shape function for several days in January 2007 from data of the FORMOSAT-3/COSMIC constellation using the UPC IONEX maps as VTEC source.

was for a previously analyzed data set by Aragon-Angel et al. [2009] (more than 17,000 solved radio occultations). The histogram (Figure 11) with the value of the sum given in equation (10) was depicted for the shape functions corresponding to the derived density profiles from FORMOSAT-3/ COSMIC for such data set. Most of the integral values were far below from the hypothetical value of one. As commented before, there are LEO satellites from the FORMOSAT-3/ COSMIC constellation at different orbits. One first interpretation of Figure 11 could be that the LEOs at lower orbital heights are the ones presenting the lower values of the integral. Nevertheless, Figure 12 does not show correlations between orbital height and integral values. Looking back at Figure 11, an underdetermination of the integral values is evident.

[28] Trying to understand the two modal distribution of the values of the integrals of the shape function in Figure 11, they were plotted versus latitude. A clear marked latitudinal dependence was evidenced (Figure 13).

[29] All these dependences become clear once the integral value of the shape functions is depicted versus time (see Figure 14). The lowest values of the integral correspond to nighttime while the highest ones, to daytime. Since the plasmaspheric contribution is more important during night, the corresponding fraction of VTEC becomes smaller during

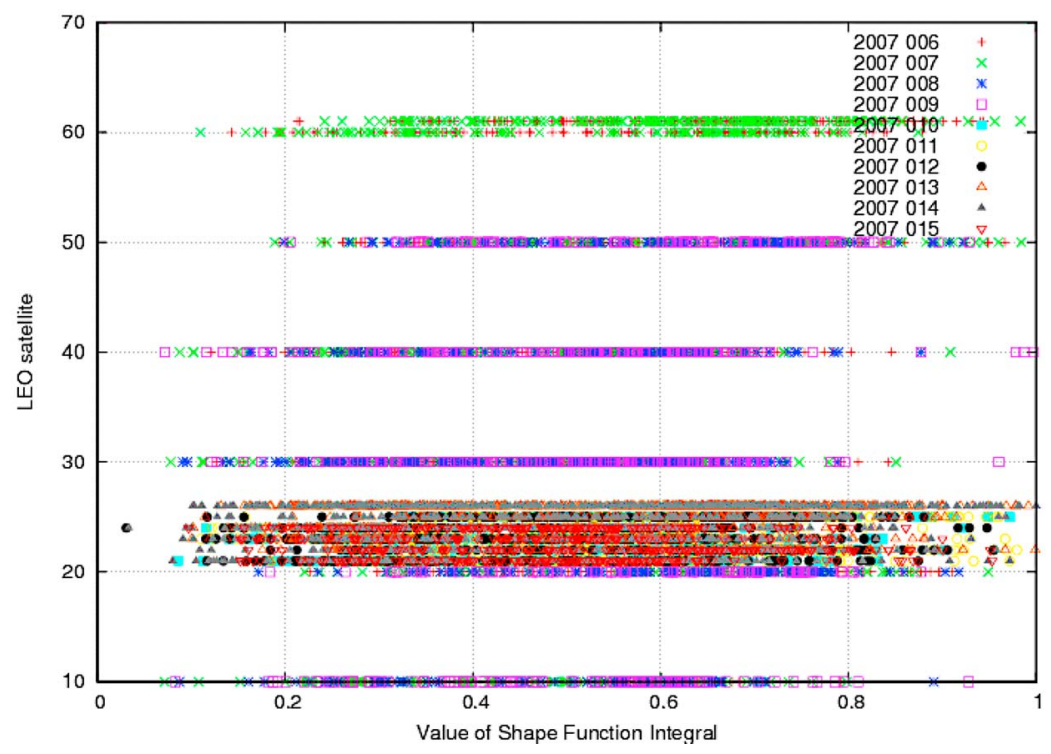

Figure 12. Histogram of the values of the integral of the shape function with respect the different LEO heights in the FORMOSAT-3/COSMIC constellation for several days in January 2007. 


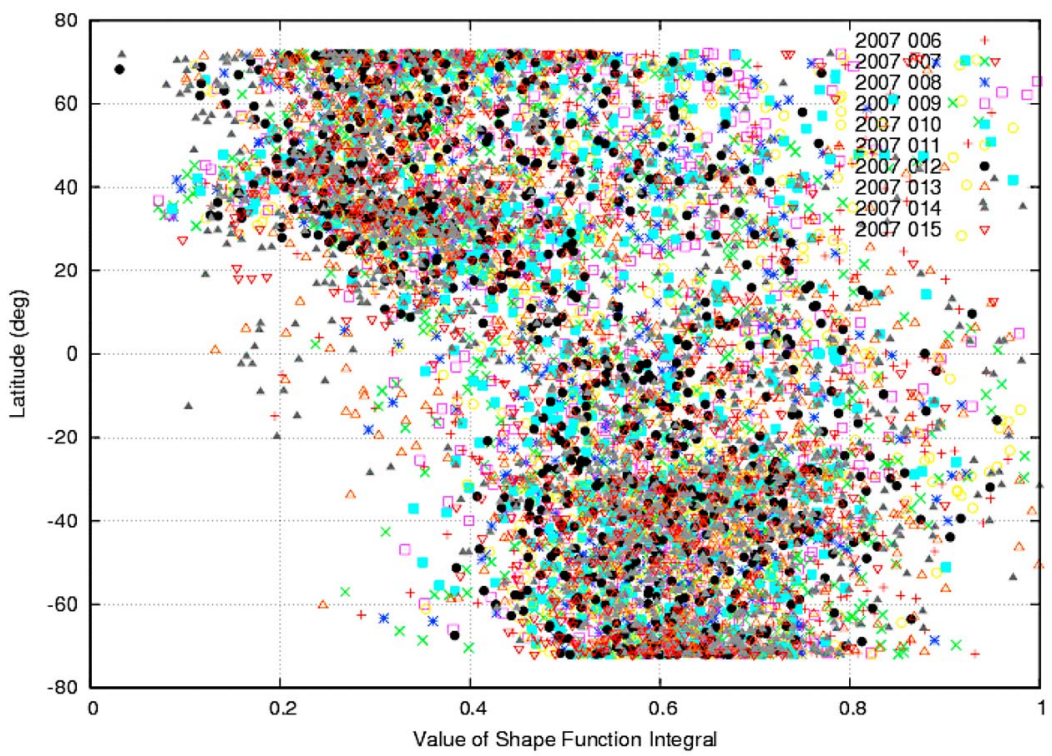

Figure 13. Comparative of the values of the integral of the shape function versus latitude for several days in January 2007. There is a marked latitudinal dependence evidencing the two modal distribution of the values of the shape function found in Figure 11.

night rather than day. Therefore, one likely interpretation would be that the integral values of the shape functions can provide a direct comparison between the VTEC derived from GPS observations and the VTEC derived from radio occultation data. Consequently, radio occultation observations are mainly sensitive to the ionosphere, not accounting for the plasmaspheric contribution. Notice that, in this implementation, the VTEC variations are driven by the IGS ionospheric maps, which are obtained from GPS measurements. Unfortunately, there is a lack of receivers in the equatorial area, where the VTEC gradients are expected to be higher. In these areas, the VTEC values are basically obtained from extrapolation techniques, producing a higher smoothed VTEC than the real one. Moreover, also notice that the procedure used in this paper involves differences between consecutive observations. Therefore, this procedure will be more sensitive to structures with higher spatial/ temporal variations, as it is expected for the ionosphere in comparison with the plasmasphere. In this sense, what we are mainly seeing in the RO retrieval is the ionospheric

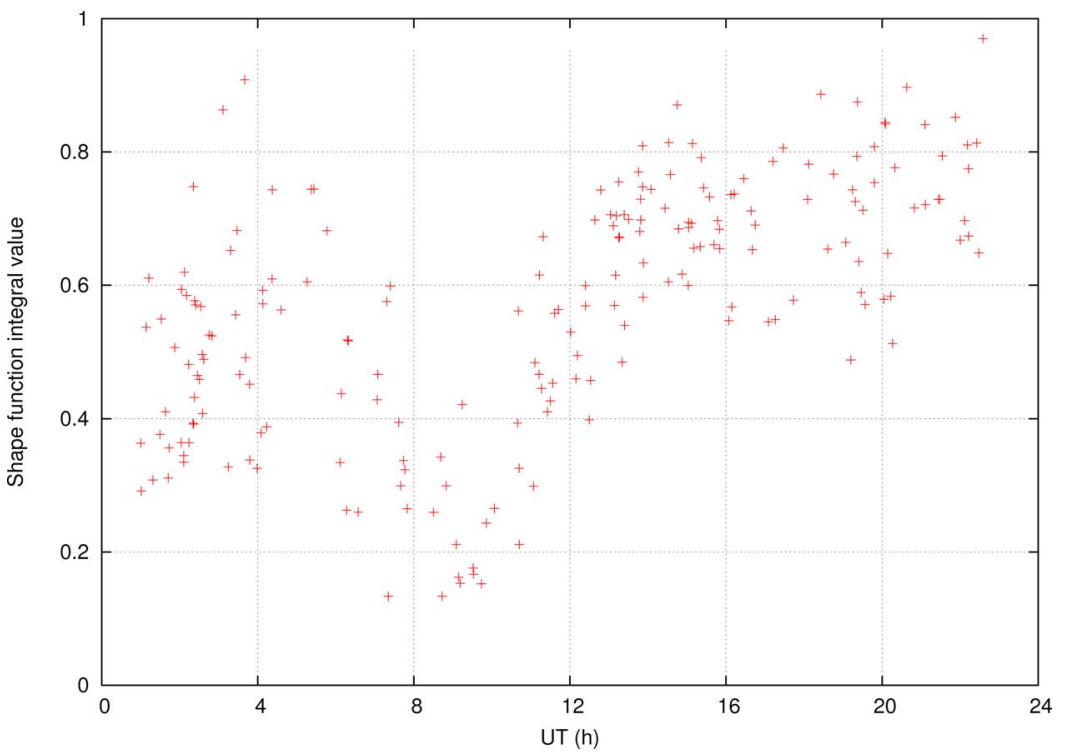

Figure 14. Integral of shape functions depicted versus universal time (UT) at Jicamarca: In general, lower values of the integral correspond to nighttime whereas higher values correspond to daytime. Notice the V pattern: when the source of ionization is down the horizon, the values start to drop to the lowest values, and then they start increasing again at dawn. 
contribution, while the IGS VTEC corresponds to both ionosphere and plasmasphere.

\section{Conclusions}

[30] Procedures to retrieve electron density profiles with high resolution and low computational burden, which were developed during previous LEO GPS occultation missions such as GPS/MET, SAC-C and CHAMP, have proven their validity with FORMOSAT-3/COSMIC constellation data as well. The use of the separability hypothesis in the Abel transform inversion, which models horizontal gradients, provides an improvement with respect to classical methods, even under high variable ionospheric conditions, such as the ones found in the neighboring area of Jicamarca for which a 1 year experiment has been carried out using as reference data manually calibrated Jicamarca DPS measurements.

[31] Under very strict colocation criteria around Jicamarca DPS location, an exhaustive comparison of the classical Abel and the improved Abel inversion has been carried out, showing that the separability concept can help correcting about $10 \%$ the error due to the spherical symmetry assumption used in the classical Abel inversion. Although previous studies showed improvements up to $45 \%$ when using separability, it must be pointed out that one of the main sources of error is the colocation of the derived profiles and the reference. In this data set, since this effect has been highly mitigated, it explains the lower percentage of improvement compared to previous results. Actually, this means that it is the shape function that helps spreading the basic information that would help recovering a density profile at any other surrounding location.

[32] Two schemes to account for the upper electron content have been implemented to both, classical and improved Abel inversions: The use of a climatological model and an exponential decay. They show an improvement with respect the use of the assumption of the refractive index to be 1 at the LEO and GPS position.

[33] When trying to validate the separability hypothesis, some inconsistencies have been found leading to the necessity for further improvement to account for the upper plasmaspheric contribution when deriving the total electron content contribution: in spite of being a small (but constant) contribution, it should be accounted for specially when the ionospheric TEC is small.

[34] A revision of the diurnal slab thickness in the models used for the topside modeling of the DPS reconstructed profiles is recommended.
[35] Acknowledgments. The authors would like to express their gratitude to the University Corporation for Atmospheric Research (UCAR) and the National Space Organization (NSPO) in Taiwan for the availability of FORMOSAT-3/COSMIC constellation data, as well as the International GNSS Service (IGS) for making available IONEX files. Angela Aragon-Angel and Manuel Hernandez-Pajares thank NSPO for their respective research fellowships to visit the Center for Space and Remote Sensing Research at the National Central University. This work has been partially supported by the Spanish Ministry of Science and Technology and the European Social Funds under the program "Personal Tecnico de Apoyo," the projects ESP2007-62676 and CTM2010-21312-C03-0.

\section{References}

Aragon-Angel, A., M. Hernández-Pajares, J. M. Juan, and J. Sanz (2009), Obtaining more accurate electron density profiles from bending angle with GPS occultation data: FORMOSAT-3/COSMIC constellation, Adv. Space Res., 43(11), 1694-1701.

Davies, K. (1990), IEE Electromagn. Waves Ser., vol. 31, Ionospheric Radio, chap. 5, pp. 124-154, Peter Peregrinus, Herts, U. K., ISBN:086341186X

Fjeldbo, G., A. Kliore, and V. Eshelman (1971), The neutral atmosphere of venus as studied with the Mariner V radio occultation experiments. Astron. J., 76, 123-140.

Hajj, G. A., and L. J. Romans (1998), Ionospheric electron density profiles obtained with the Global Positioning System: Results from the GPS/MET experiment, Radio Sci., 33(1), 175-190.

Hernández-Pajares, M., J. M. Juan, J. Sanz, and J. G. Sole (1998), Global observation of the ionospheric electronic response to solar events using ground and LEO GPS data, J. Geophys. Res., 61, 1237-1247.

Hernández-Pajares, M., J. M. Juan, and J. Sanz (2000), Improving the Abel inversion by adding ground data LEO radio occultations in the ionospheric sounding, Geophys. Res. Lett., 27, 2743-2746.

Jakowski, N., A. Wehrenpfennig, S. Heise, C. Reigber, H. Lühr, L. Grunwaldt, and T. K. Meehan (2002), GPS radio occultation measurements of the ionosphere from CHAMP: Early results, Geophys. Res. Lett., 29(10), 1457, doi:10.1029/2001GL014364.

Jakowski, N., A. Wehrenpfennig, S. Heise, C. Reigber, and H. Lühr (2003), Status of ionospheric radio occultation CHAMP data analysis and validation of higher level data products, in First CHAMP Science Mission Results for Gravity, Magnetic and Atmospheric Studies, pp. 462-472, Springer, Berlin, ISBN:3-540-00206-5.

Reinisch, B. W., V. V. Paznukhov, I. A. Galkin, D. Altadill, and J. McElroy (2008), Precise radar range measurements with digisondes, AIP Conf. Proc., 974, 144-149, doi:10.1063/1.2885023.

Rocken, C., Y. H. Kuo, W. Schreiner, D. Hunt, and S. Sokolovskiy (2000), COSMIC system description, Atmos. Oceanic Sci., 11(1), 21-52.

Schreiner, W. S., S. V. Sokolovskiy, and C. Rocken (1999), Analysis and validation of GPS/MET radio occultation data in the ionosphere, Radio Sci., 34(4), 949-966.

Tricomi, F. G. (1985), Integral Equations, Dover Publ., New York.

$\mathrm{Wu}, \mathrm{X}$., X. Hu, X. Gong, X. Zhang, and X. Wang (2009), Analysis of inversion errors of ionospheric radio occultation, GPS Solutions, 13, 231-239.

A. Aragon-Angel, M. Hernández-Pajares, M. Juan, and J. Sanz, Research Group of Astronomy and Geomatics, Technical University of Catalonia, Mod. C3 Campus Nord UPC, Jordi Girona 1, Barcelona E-08034, Spain. (angela@ma4.upc.edu)

C.-C. Lee, General Education Center, Ching-Yun University, 229 ChienHsin Rd., Jhongli 32097, Taiwan.

Y.-A. Liou, Center for Space and Remote Sensing Research, National Central University, 300 Jhongda Rd., Jhongli 32001, Taiwan.

B. W. Reinisch, Center for Atmospheric Research, University of Massachusetts, 600 Suffolk St., Lowell, MA 01854, USA. 